\title{
Topography and Morphology of a Hydrogel Enriched with Mimosa tenuiflora for Tissue Regeneration
}

Rosa Saucedo-Acuña ${ }^{1}$, Karla Gutiérrez-Holguín ${ }^{1}$, Karla Tovar-Carrillo ${ }^{1}$, Guillermina Martínez-Moreno ${ }^{1}$ and Carlos Rodríguez-Rodríguez ${ }^{2}$

${ }^{1}$ Instituto de Ciencias Biomédicas - Universidad Autónoma de Ciudad Juárez, Juarez, Chihuahua, Mexico, ${ }^{2}$ Universidad Tecnológica de Ciudad Juárez, Juarez, Chihuahua, Mexico

The main purpose for preparation of PVA (polyvinyl alcohol) hydrogel enriched with Mimosa tenuiflora is to offer a new alternative in tissue regeneration field. In this manner, PVA confers soft consistency to hydrogel matrix and allows homogeneous distribution of mimosa in the material. Several approaches reported different uses for PVA in pharmaceutical, biomedical and other fields. The biomedical applications of PVA include eye drops, contact lenses, artificial cartilage, and more, due to its softness, roughness and porosity [1-6]. In addition, PVA is being used to elaborate controlled interconnected micro porous three dimensional (3D) scaffolds for various applications, making PVA an excellent substitute of conjunctive tissue.

Moreover, Mimosa tenuiflora is a commonly fond weed which is easily dismissed as an invasive plant with significant history in several traditional medicinal systems as a potent antioxidant, anti-inflammatory and antimicrobial properties [7-9]. Mimosa tenuiflora is native to south and central America and is also cultivated in India. Among all, reports showed wound healing activity of Mimosa tenuiflora in wound models. With the combination of both components in the hydrogel, a material with excellent properties for medical applications could be obtained with the main healing purpose by offering a suitable environment for mimosa release into the wound.

Due to PVA and mimosa properties and purpose of the obtained hydrogel, surface analyze was made. It is well known that materials surface properties play on important role in tissue regeneration and determine cell adhesion and proliferation given a key point for wound healing process.

For the image analysis, a JEOL microscope model JSM-6010PLUS/LA was used. Samples within a size of 5 x $5 \mathrm{~mm}$ were dried $24 \mathrm{~h}$ in vacuum. Images were taken at low vacuum at $10 \mathrm{kV}$. To obtain a closer image of the hydrogel enriched with the extract, we cover the sample with gold.

Figure 1 shows internal longitudinal section of the surface of the hydrogel and Figure 2 shows the external longitudinal section of the hydrogel. Hydrogel shows two different longitudinal faces as result of the molding process where the softness material is dried.

In all cases, the hydrogel shows a homogeneous and roughness surface, even keeping micro and nano porous. We can verify too, the structure of the Mimosa particles not reported before. As conclusion, the idea of enriched a PVA hydrogel with Mimosa tenuiflora give to the hydrogel an advantage for biomedical proposes. 

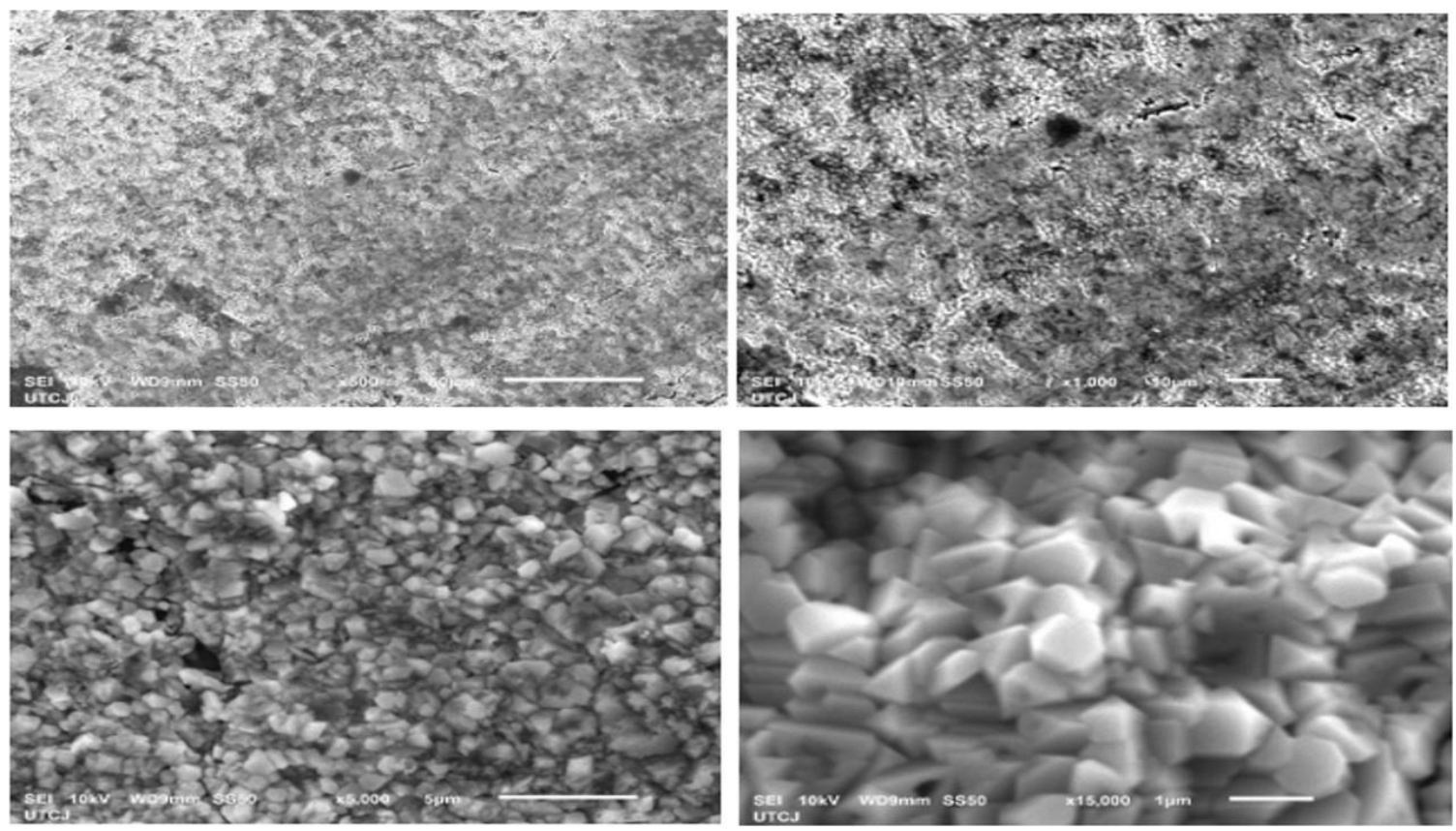

Figure 1. Internal longitudinal surface of the hydrogel enriched in Mimosa at 500, 1000, 5000 and 15000x respectively.
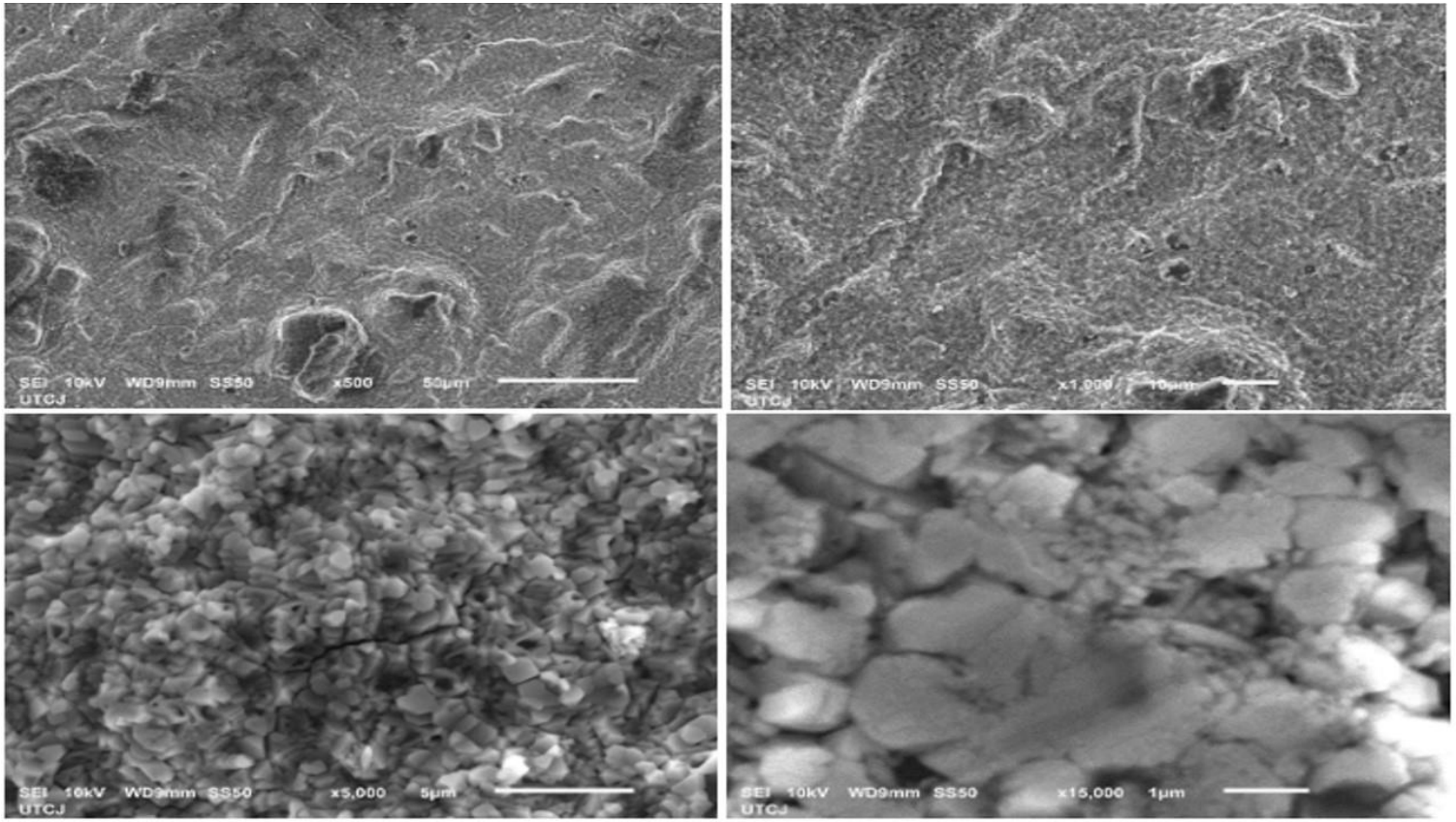

Figure 2. External longitudinal surface of the hydrogel enriched in Mimosa at 500, 1000, 5000 and 15000x respectively.

\section{References}

[1] J. Dow Biomacromolecules, 19(1) (2018) p. 158

[2] Q. Ding, et. al., ACS Appl. Mater. Interfaces 10(33) (2018), p. 27987

[3] L. Coluccino, et. al., ACS Biomaterials Science \& Engineering, 4(5) (2018), p. 1518

[4] M. Rizwan, et. al., ACS Applied Biomaterials 3(1) (2020), p. 693

[5] H. Zhang, H. Xia, and Y. Zhao. ACS Macro Letters 1(11) (2012), p. 1233 
[6] A. Kumar, T. Behl, and S. Chadha. Internat. J. of Biological Macromolecules 149 (2020), p.1262

[7] L. E. Valencia-Gómez, et. al., Internat. J. of Biological Macromolecules 93A (2016), p.1217

[8] H. Jian, K. Tovar-Carrillo, and T. Kobayashi. Ultrasonics Sonochemistry 32 (2016), p. 398

[9] J. Choi, et. al., Biomedicine \& Pharmacotherapy 106 (2018), p. 326.

[10] S. Bi, et. Al., Internat. J. of Biological Macromolecules 146 (2020), p. 99 